\title{
A universal multi-dimensional charge and mass transfer model
}

\author{
G. Kennell \& R. W. Evitts \\ Department of Chemical Engineering, University of Saskatchewan, \\ Canada
}

\begin{abstract}
A new approach has been developed for modelling the transport of charge and mass through electrolytes. This approach utilizes a dynamically computed electric field that contributes to the transport of charged species in much the same way a flow field contributes to the transport of mass. Since a multidimensional electric field is computed, the effects of this field on redox reactions occurring in the electrolyte or at the interface of an electronic and ionic conductor can be modelled. Transport of species due to diffusion and convection is also considered, and the overall transport system is modelled using a control volume technique with a modified Peclet number. Electroneutrality and the conservation of species are incorporated into the model. This method produces a universal model capable of predicting one, two, or three-dimensional mass and charge transport for electrochemical phenomena where macroscopic anodic and cathodic couples exist. This model has the potential to simulate forms of localized corrosion and energy storage and generation applications such as batteries and fuel cells. The theoretical development and validation of this new model for a two dimensional case study is presented.
\end{abstract}

Keywords: electrochemistry, modelling, computational fluid dynamics, control volume technique, polarization, electric field, electrolyte.

\section{Introduction}

The open literature contains many articles on modelling of charge and mass in numerous electrochemical systems. Some examples of these systems include: fuel cells, batteries, pitting corrosion, and crevice corrosion. In such systems the transport of charged species is usually significantly affected by the electric field. 
However, the transport of charged species can also affect the electric field, which in turn affects the further transport of charged species. In crevice corrosion many important phenomena depend upon the electric field caused by the transport (or resistance to transport) of charged ions between predominantly anodic and cathodic areas. Even the rate of electrochemical reactions occurring at cathodic and anodic areas has been shown to be dependent on the electric field [1]. The methods presented in this paper represent the development of a multidimensional model that simultaneously considers the dynamic electric field, its affect on transport and redox reaction kinetics, and the interaction of these with the field.

One of the major applications of this model is the simulation of crevice corrosion. This model has been developed to simulate crevice corrosion by considering not only the crevice, but also the coupled reactions occurring along the bold surface, using a single set of equations that spans the whole computational domain. However, the application of this multi-dimensional model is not limited to localized forms of corrosion. Although not presented here, the method outlined in this paper may be applied to model crevice corrosion and other electrochemical systems, such as batteries and fuel cells.

\section{Modelling transport and the electric potential field}

The model utilizes the comprehensively developed control volume technique described by Patankar [2]. Infinitely dilute solution theory is used as the basis of the equation describing the electric field. Two main equations are numerically solved for the multi-dimensional transport:

$$
\frac{\partial C_{i}}{\partial t}-\left(z_{i} u_{i} \mathrm{~F} \nabla \boldsymbol{\Phi}-\mathbf{V}\right) \nabla C_{i}=D_{i} \nabla^{2} C_{i}+S_{i},
$$

and

$$
\nabla^{2} \boldsymbol{\Phi}=-\frac{\mathrm{F}}{\kappa} \sum_{i} z_{i} D_{i} \nabla^{2} C_{i}-\frac{\mathrm{F}}{\kappa} \sum_{i} z_{i} S_{i}-\frac{\mathrm{F}}{\kappa} \sum_{i} z_{i} \frac{C_{i}}{\partial t} .
$$

Eqn (1) describes the transport of charged species due to concentration and potential gradients. Eqn (2) describes the electric field established due to concentration gradients of charged species (diffusion potential), physically separated but electrically conserved anodic and cathodic reactions, and electroneutrality. These two equations are solved independently. Eqn (1) is solved utilizing established techniques [2], and the development of its numerical representation is shown in Section 2.1. Eqn (2) is solved using the AlternatingDirection Implicit Method, although other methods could be used. During the numerical solution of eqn (1) a differencing scheme, such as The Upwind Scheme, The Hybrid Scheme, or The Power-Law Scheme, utilizes a Peclet number [2]. (The Power-Law Scheme was utilized during the numerical predictions presented in this paper.) However, since the Peclet number does not consider the transport of species due to migration, a new Peclet number is 
defined. This Peclet number is similar to the Peclet number proposed by Heppner [3], but also considers convection, as well as migration and diffusion:

$$
P=\frac{\left(z_{i} u_{i} \mathrm{~F} \nabla \boldsymbol{\Phi}-\mathbf{V}\right) \Delta x}{D_{i}} .
$$

Similar to eqn (1), the migration term in eqn (3) acts as an amendment to the flow field that is specific to the charge and mobility of each species. This does not significantly increase the complexity of the numerical solution of eqns (1) or (3).

\subsection{Solving the transport equation}

The development of the two-dimensional transport model is shown below. Effort has been taken to present this development and associated nomenclature in a manner that corresponds to that described by Patankar [2]. This new transport model (easily capable of being developed in three dimensions) is particularly easy to solve using numerical methods due to the manner in which the flux is integrated over each control volume. This integration method maintains the expression describing the migration of ions in its simplest form with only two variables to be solved for: the concentration of species and gradient of potential. The term describing the migration of ions is the first term on the right hand side of eqn (4). The flux of each dissolved species under the influence of a potential field (assuming infinitely dilute solution theory) can be given as [4]:

$$
\mathbf{N}_{i}=-z_{i} u_{i} \mathrm{~F} C_{i} \nabla \mathbf{\Phi}-D_{i} \nabla C_{i}+C_{i} \mathbf{V}
$$

The subscript, $i$, indicating an individual species is dropped in the following development for clarity. It is, however, assumed that each of the following equations in this sub-section can be written for each dissolved species. From eqn (4) it can be seen that the flux in the $x$-dimension and $y$-dimension can be expressed by:

$$
\begin{aligned}
& J_{x} \equiv\left(U-z u \mathrm{~F} \frac{d \boldsymbol{\Phi}}{d x}\right) C-D \frac{d C}{d x}, \\
& J_{y} \equiv\left(V-z u \mathrm{~F} \frac{d \boldsymbol{\Phi}}{d y}\right) C-D \frac{d C}{d y}
\end{aligned}
$$

and the integration of eqn (4) gives:

$$
\frac{\left(C_{P}-C_{P}^{0}\right) \Delta x \Delta y}{\Delta t}+J_{n}+J_{e}-J_{s}-J_{w}=\left(S_{c}+S_{p} C_{P}\right) \Delta x \Delta y .
$$


Eqn (7) is the equation developed in Patankar [2] for convective-diffusive problems. However, in this paper the fluxes in eqn (7) include the migration terms expressed by eqns (5) and (6). Thus, the numerical techniques that have evolved during the development of the Control Volume method may be utilized to solve convective-diffusive problems with electromigration. Eqn (7) is the discretized form of eqn (1). The mass flow rates through the faces of a control volume are given by:

$$
\begin{aligned}
& F_{n}=\left(V-z u \mathrm{~F} \frac{d \boldsymbol{\Phi}}{d y}\right)_{n} \Delta x, \\
& F_{s}=\left(V-z u \mathrm{~F} \frac{d \boldsymbol{\Phi}}{d y}\right)_{s} \Delta x, \\
& F_{e}=\left(U-z u \mathrm{~F} \frac{d \boldsymbol{\Phi}}{d x}\right)_{e} \Delta y, \\
& F_{w}=\left(U-z u \mathrm{~F} \frac{d \boldsymbol{\Phi}}{d x}\right)_{w} \Delta y .
\end{aligned}
$$

The migration term in the current development occurs as an amendment to the flow field term. The addition of these two terms creates a flow field that does not satisfy the volume continuity equation for the case of incompressible flow. This is realistic when considering the migration of a single species. However, conservation of species due to transport is assured in this model because any flux leaving a control volume is the flux entering a neighbouring control volume. This is a property of the Control Volume technique. For a flow field that does not satisfy the volume continuity equation, eqn (7) can be rearranged to be:

$$
\begin{aligned}
& \frac{\left(C_{P}-C_{P}^{0}\right) \Delta x \Delta y}{\Delta t}+\left(F_{n}+F_{e}-F_{s}-F_{w}\right) C_{P}+\left(J_{n}-F_{n} C_{P}\right) \\
& +\left(J_{e}-F_{e} C_{P}\right)-\left(J_{s}-F_{s} C_{P}\right)-\left(J_{w}-F_{w} C_{P}\right), \\
& =\left(S_{c}+S_{p} C_{P}\right) \Delta x \Delta y \\
& \frac{\left(C_{P}-C_{P}^{0}\right) \Delta x \Delta y}{\Delta t}+\left(F_{n}+F_{e}-F_{s}-F_{w}\right) C_{P}+a_{N}\left(C_{P}-C_{N}\right) \\
& +a_{E}\left(C_{P}-C_{E}\right)-a_{S}\left(C_{S}-C_{P}\right)-a_{W}\left(C_{W}-C_{P}\right), \\
& =\left(S_{c}+S_{p} C_{P}\right) \Delta x \Delta y \\
& a_{P} C_{P}=a_{N} C_{N}+a_{E} C_{E}+a_{S} C_{S}+a_{W} C_{W}+b,
\end{aligned}
$$


where:

$$
\begin{gathered}
a_{P}=\left(\frac{\Delta x \Delta y}{\Delta t}+F_{n}+F_{e}-F_{s}-F_{w}+a_{N}+a_{E}-a_{S}-a_{W}+S_{P} \Delta x \Delta y\right) \\
a_{N}=D_{n} A\left(\left|P_{n}\right|\right)+\left[\left[-F_{e}, 0\right]\right] \\
a_{E}=D_{e} A\left(\left|P_{e}\right|\right)+\left[\left[-F_{e}, 0\right]\right] \\
a_{S}=D_{s} A\left(\left|P_{s}\right|\right)+\left[\left[F_{s}, 0\right]\right] \\
a_{W}=D_{w} A\left(\left|P_{w}\right|\right)+\left[\left[F_{w}, 0\right]\right]
\end{gathered}
$$

and

$$
b=\left(S_{c}+\frac{C_{P}^{0}}{\Delta t}\right) \Delta x \Delta .
$$

The calculation of the function, $A\left(\left|P_{n}\right|\right)$, depends on the differencing scheme as indicated earlier in this paper.

\subsection{Development of an equation for the electric potential field}

The following is the development of an equation that describes the electric field in the electrolyte. This equation does not include effects very close to the electrode surface in the charge double layer. A material balance for a single species shows that the change in concentration of a species depends on the divergence (due to flux) and production of that species [4]:

$$
\frac{\partial C_{i}}{\partial t}=-\nabla \cdot \mathbf{N}_{i}+S_{i}
$$

Multiplying eqn (21) by $z_{i} \mathrm{~F}$ gives:

$$
\frac{\partial}{\partial t}\left(\mathrm{~F} \sum_{i} z_{i} C_{i}\right)=-\nabla \cdot \mathrm{F} \sum_{i} z_{i} \mathbf{N}_{i}+\mathrm{F} \sum_{\mathrm{i}} z_{i} S_{i} .
$$

This model is designed to predict concentration profiles that are electrically neutral. The electroneutrality assumption has been shown to be a very good approximation of reality [4]: 
186 Advances in Fluid Mechanics VIII

$$
\sum_{i} z_{i} C_{i}=0
$$

Substituting eqn (23) into eqn (22) gives eqn (24).

$$
\nabla \cdot \mathrm{F} \sum_{i} z_{i} \mathbf{N}_{i}=\mathrm{F} \sum_{i} z_{i} S_{i}
$$

Current in an electrolyte solution is:

$$
\mathbf{i}=\mathrm{F} \sum_{i} z_{i} \mathbf{N}_{i}
$$

Substituting eqn (25) into eqn (24) gives:

$$
\nabla \cdot \mathbf{i}=\mathrm{F} \sum_{i} z_{i} S_{i}
$$

Eqn (26) implies there is no divergence of electrical current except for the transport of charge created by physically separated anodic and cathodic reactions. Current density is given by [4]:

$$
\mathbf{i}=-\mathrm{F}^{2} \nabla \boldsymbol{\Phi} \sum_{i} z_{i}^{2} u_{i} C_{i}-\mathrm{F} \sum_{i} z_{i} D_{i} \nabla C_{i}+\mathrm{Fv} \sum_{i} z_{i} C_{i}
$$

From eqn (27), the divergence of current is therefore:

$$
\nabla \cdot \mathbf{i}=-\nabla \cdot(\kappa \nabla \boldsymbol{\Phi})-\mathrm{F} \sum_{i} z_{i} \nabla \cdot D_{i} \nabla C_{i}+\mathrm{Fv} \sum_{i} z_{i} C_{i}
$$

where the conductivity is:

$$
\kappa=\mathrm{F}^{2} \sum_{i} z_{i}^{2} u_{i} C_{i}
$$

The last term of eqn (28) is zero since the system is assumed to be electrically neutral. Substituting eqn (26) into eqn (28) and assuming constant conductivity and diffusion coefficients over a control volume yields an equation that describes the electrical field:

$$
\nabla^{2} \boldsymbol{\Phi}=-\frac{\mathrm{F}}{\kappa} \sum_{i} z_{i} D_{i} \nabla^{2} C_{i}-\frac{\mathrm{F}}{\kappa} \sum_{i} z_{i} S_{i} .
$$




\subsection{Electroneutrality}

An analogous equation to eqn (1) can be derived as follows; substituting eqn (4) into eqn (21) gives:

$$
\frac{\partial C_{i}}{\partial t}=-\nabla \cdot\left(-z_{i} u_{i} \mathrm{~F} C_{i} \nabla \mathbf{\Phi}-D_{i} \nabla C_{i}+C_{i} \mathbf{V}\right)+S_{i} .
$$

Using the chain rule to differentiate eqn (31) and rearranging:

$$
\frac{\partial C_{i}}{\partial t}-\left(z_{i} u_{i} \mathrm{~F} \nabla \boldsymbol{\Phi}-\mathbf{V}\right) \nabla C_{i}=D_{i} \nabla^{2} C_{i}+S_{i}+z_{i} u_{i} \mathrm{~F} \nabla^{2} \mathbf{\Phi} C_{i} .
$$

Comparison of eqn (32) with eqn (1) shows that there is an additional term on the right hand side of eqn (32). The absence of this term in eqn (1) is one of the inherent strengths of the model presented in this paper. Numerically, this extra term is a species source term dependent on charge density. This previous statement is made assuming a more rigorous definition of near-electroneutrality for a medium with uniform dielectric constant [4]:

$$
\nabla^{2} \Phi=-\frac{\mathrm{F}}{\varepsilon} \sum_{i} z_{i} C_{i}
$$

If eqn (32) was solved using the actual potential field in the system, and no errors were introduced during the numerical procedure, conservation of species should occur; however, the guarantee of species conservation for all situations (assured for the model presented in this paper) is not evident for the numerical solution of eqn (32) i.e., when rounding errors occur.

There is one disadvantage of the new model. In deriving eqn (30) electroneutrality was assumed. However, discretization of eqns (1) and (30) will introduce errors in their solution and therefore there is no guarantee of electroneutrality. A predicted concentration that differs significantly from electroneutrality would be unreasonable. However, if the derivation presented in section 2.2 is repeated with all affects on the Laplacian of the electric field neglected except for non-electroneutrality (in the current model any nonelectroneutrality would be caused by numerical error):

$$
\nabla^{2} \boldsymbol{\Phi}=\frac{\mathrm{F}}{\kappa} \sum_{i} z_{i} \frac{\partial C_{i}}{\partial t}
$$

Therefore, the Laplacian of the electric potential expressed in eqn (34) may be thought of as a representation of the numerical error generated over the previous time step. Then the Laplacian of the electric potential needed to cause the 
transport of charge that will eliminate any error (in regards to electroneutrality) that has occurred up to the current time step over the next time step is:

$$
\nabla^{2} \boldsymbol{\Phi}=-\frac{\mathrm{F}}{\kappa} \sum_{i} z_{i} \frac{C_{i}}{\partial t}
$$

Adding eqn (35) to eqn (30) yields eqn (2). Thus, eqn (2) ensures an electrically neutral system is predicted. Eqn (2) is also generally not a stiff equation, unlike eqn (33), due to the significantly larger value of conductivity when compared with the dielectric constant, for most systems.

\subsection{Boundary conditions}

For the numerical solution of eqns (1) and (2) boundary conditions are needed. Three situations existing at the boundary have been considered for the simulation presented in Section 3: bulk electrolyte, a barrier through which no mass or charge is transported, or a barrier through which charge and mass is only transported via an electrochemical reaction (i.e. an electroactive surface). For the situation that involves bulk electrolyte the boundary condition for eqn (1) may be given as

$$
C_{i}=A
$$

and for eqn (2)

$$
\boldsymbol{\Phi}=B
$$

For the situation where a barrier through which no charge or mass is transported exists at the boundary, the boundary condition for eqn (1) is

and for eqn (2) is

$$
\frac{\partial C_{i}}{\partial x}=0
$$

$$
\frac{\partial \Phi}{\partial x}=0
$$

For the situation where an electroactive area is present at the boundary, the boundary condition for eqn (1) is eqn (38), and for eqn (2) it is eqn (37).

\section{Validation and discussion}

The model presented in this paper was used to model the moving boundary experiment of $\mathrm{Fu}$ and $\mathrm{Chan}$ [5]. The experiment consisted of a small diameter glass tube filled with $0.1 \mathrm{M} \mathrm{KNO}_{3}$. One end of the tube was open to a beaker containing a platinum cathode and the other end of the tube was plugged with an 
Ag anode. Upon application of a $1 \mathrm{~mA}$ current silver ions were dissolved into the electrolyte and were transported along the glass tube. During this process a transition zone was formed between areas containing mostly $\mathrm{Ag}^{+}$or $\mathrm{K}^{+}$. This transition zone, or boundary, was made visible by a small addition of ascorbic acid and its progression along the glass tube was measured. Fig. 1 shows this experimental data along with the movement of the boundary predicted by the current model and an excellent fit is evident. For this simulation 200 nodes in the direction of migration and 10 nodes in the radial direction were used along with a time step of $0.001 \mathrm{~s}$. The width of a control volume was $0.0002 \mathrm{~m}$ in both directions. A grid dependency study has been completed. The numerical model is stable when simulating using a coarser spatial and temporal grid; however, as the grid size is increased the rate of predicted transport also increases. For simulations conducted using a finer spatial and temporal grid than the validation simulation no change in the rate of transport was evident and the results corresponded with the validation results.

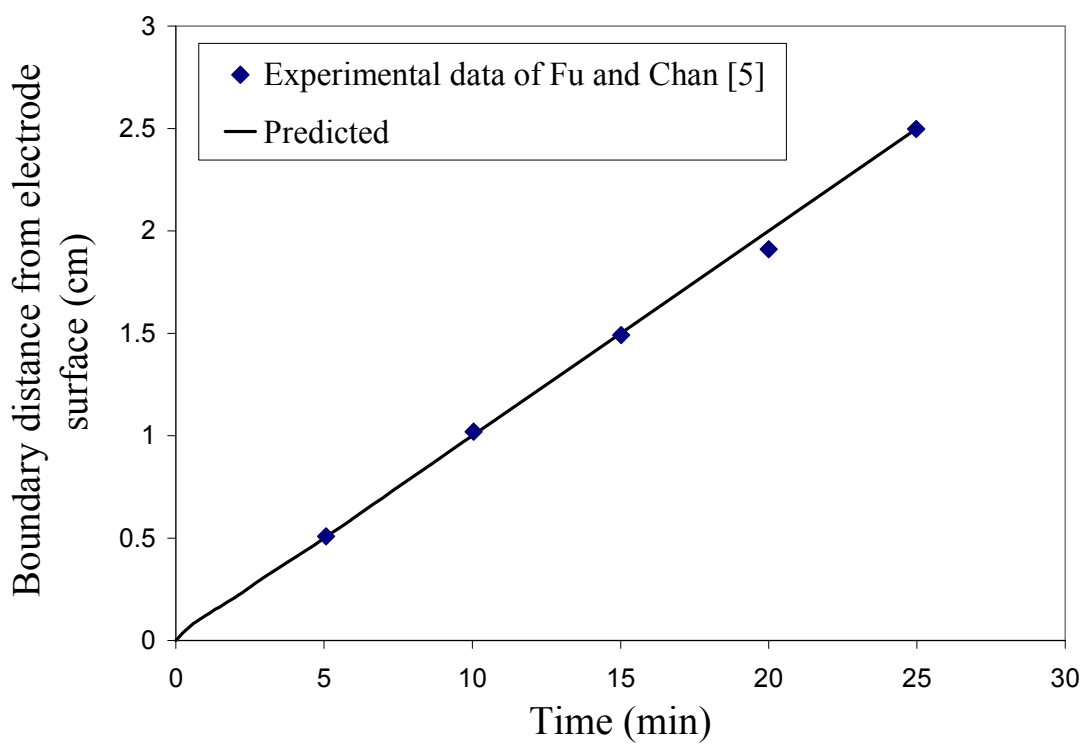

Figure 1: $\quad$ Experimentally determined and predicted boundary position.

The concentration of $\mathrm{Ag}^{+}$, as predicted by the model over two dimensions, can be seen in fig. 2. Although significant concentration gradients are only established along the length of the tube during the simulation, the validation is considered to be successful in two dimensions. This is because the prediction of insignificant radial concentration gradients matches experimental observations and verifies that errors did not propagate in the radial direction during the coupled solution of eqns (1) and (2). In fig. 2 the transition region, where the concentration of $\mathrm{Ag}^{+}$approaches zero, can be seen at approximately $1 \mathrm{~cm}$ from 


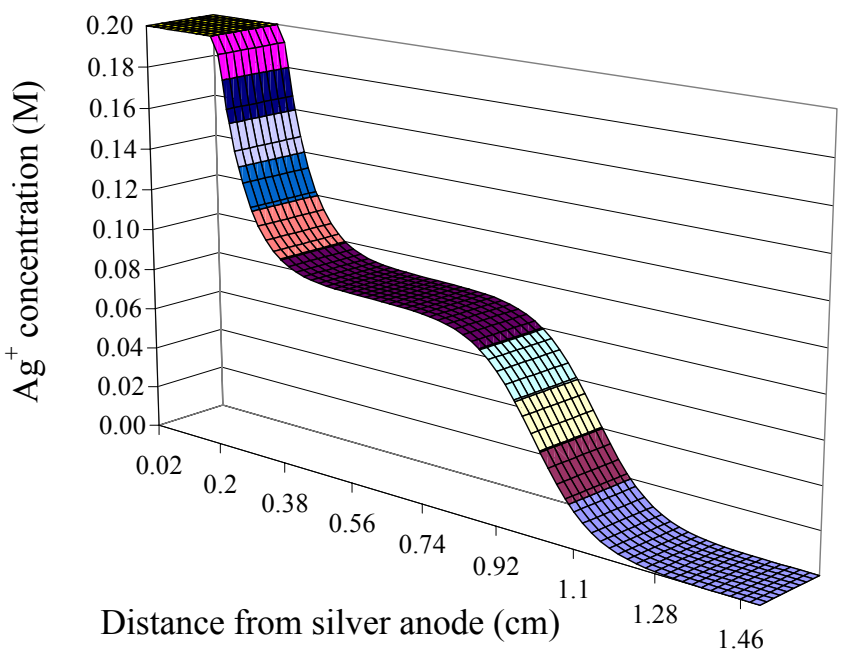

Figure 2: $\quad$ Predicted $\mathrm{Ag}^{+}$concentration after 10 mins.

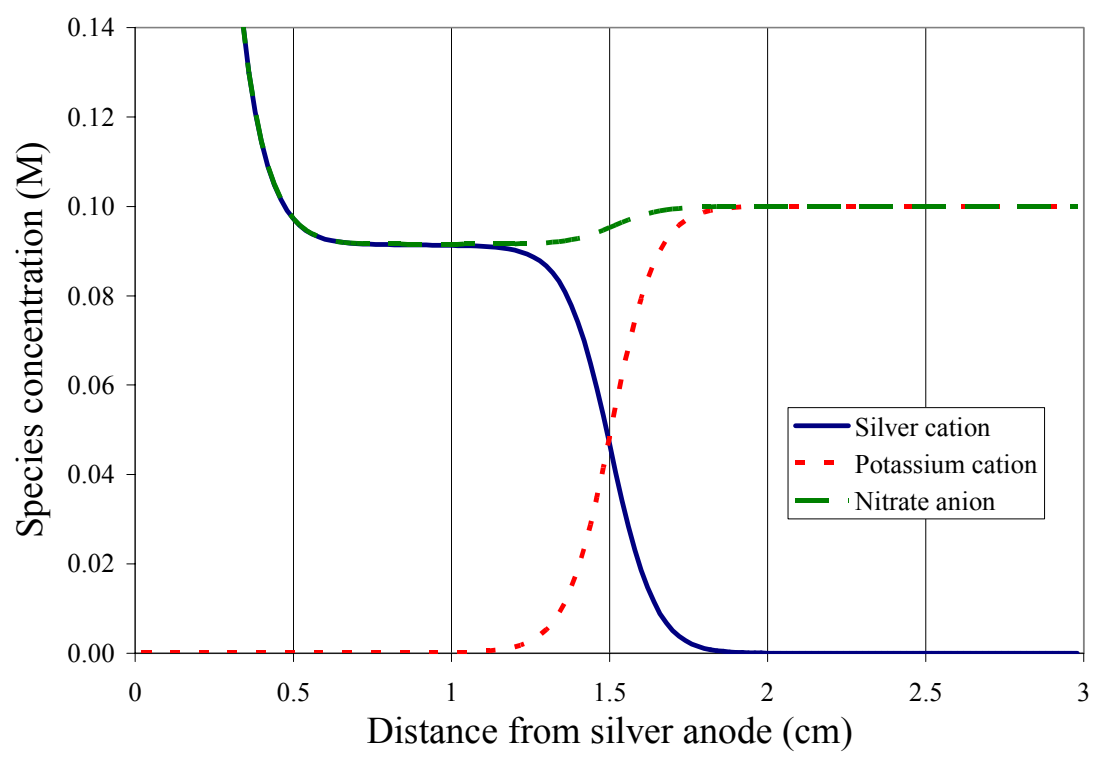

Figure 3: $\quad$ Predicted ion concentrations after 15 mins.

the silver anode. Also, a region of relatively constant concentration of $\mathrm{Ag}^{+}$exists from approximately $0.5 \mathrm{~cm}$ to the start of the transition region at about $0.75 \mathrm{~cm}$ from the anode. This region of relatively constant concentration expands in length at later times; however the concentration of $\mathrm{Ag}^{+}$in this region is steady at approximately $0.09 \mathrm{M}$. Since the model predicts no significant concentration gradients in the radial direction at any time, predicted concentrations can be 
expressed (at a set time) as a function of distance from the anode (fig. 3). Fig. 3 shows the predicted concentrations of all species in the electrolyte after 15 mins. The boundary between $\mathrm{Ag}^{+}$and $\mathrm{K}^{+}$can clearly be established at $1.5 \mathrm{~cm}$ from the anode. Fig. 3 also shows predicted concentrations that result in electroneutrality of the electrolyte and dissolved species.

It was found that the numerical solution of this model was relatively computationally inexpensive and stable when compared with other electrolyte transport models the authors have worked with $[1,6]$. A notebook PC equipped with a $1.5 \mathrm{GHz}$ Intel Celeron M processor with $504 \mathrm{MB}$ of RAM was able to solve the model presented here in approximately ten hours. Since the model is relatively computationally inexpensive it is expected that simulations times for much more complex systems will be short. This illustrates the usefulness of the methods outlined here, and the possible applicability of these methods to the transport of charge and mass through many different electrolytes in many different industrial settings.

\section{Conclusions}

A new method was presented for modelling the transport of charge and mass through electrolytes. This utilizes a control volume technique coupled with the predicted electric field. This method was validated against the experimental data of $\mathrm{Fu}$ and $\mathrm{Chan}$ [5] and excellent agreement was found. It is proposed that this new method may be useful to simulate a wide variety of electrochemical systems where multi-dimensional mass and charge transport is important, due to the stability and computational ease of the numerical solution outlined here.

\section{References}

[1] Kennell, G.F. \& Evitts, R.W., Crevice Corrosion Cathodic Reactions and Crevice Scaling Laws. Electrochimica Acta, 54, pp. 4696-4703, 2009.

[2] Patankar, S.V., Numerical Heat Transfer and Fluid Flow, Hemisphere: New York, 1980.

[3] Heppner, K.L., Development of Predictive Models of Flow Induced and Localized Corrosion, Ph.D. Thesis, University of Saskatchewan: Saskatoon, 2006.

[4] Newman, J.S., Electrochemical Systems, Prentice Hall, New Jersey, 1991.

[5] Fu, J.W. \& Chan, S., A Finite Element Method for Modeling Localized Corrosion Cells. Corrosion, 40, pp. 540-544, 1984.

[6] Kennell, G.F., Heppner, K.L., Evitts, R.W., A Critical Crevice Solution and iR Drop Crevice Corrosion Model. Corrosion Science, 50, pp. 1716-1725, 2008 . 